\title{
Téoros
}

Revue de recherche en tourisme

\section{Tourisme et régions : survol de la littérature internationale spécialisée}

\section{Daniel Dumas}

Volume 5, numéro 1, mars 1986

Tourisme et régions

URI : https://id.erudit.org/iderudit/1080596ar

DOI : https://doi.org/10.7202/1080596ar

Aller au sommaire du numéro

Éditeur(s)

Université du Québec à Montréal

ISSN

0712-8657 (imprimé)

1923-2705 (numérique)

Découvrir la revue

Citer ce document

Dumas, D. (1986). Tourisme et régions : survol de la littérature internationale spécialisée. Téoros, 5(1), 39-40. https://doi.org/10.7202/1080596ar d'utilisation que vous pouvez consulter en ligne.

https://apropos.erudit.org/fr/usagers/politique-dutilisation/ 


\section{Tourisme et régions: survol de la littérature internationale spécialisée

\author{
par Daniel Dumas *
}

Le présent numéro de Téoros illustre et documente largement le rapprochement et le lien entre tourisme et développement régional au Québec. Pour élargir la réflexion, nous vous proposons, à partir d'une sélection d'articles tirés de la littérature touristique internationale, un survol des thèmes abordés par les chercheurs. Les premiers textes, mettant clairement en relation tourisme et régions, présentent le tourisme comme un facteur d'expansion ou de valorisation régionale (Defert, 1966) (A.I.E.S.T., 1960). Nous sommes en Europe, on parle déjà de "développement régional".

Un nouveau discours $s^{\dagger}$ est élaboré. Cela s'est fait au Québec dans les années soixante. Plus tôt ou plus tard ailleurs, ce nouveau discours origine d'une prise de conscience du phénomène des disparités et des inégalités du développement. "De nouveaux concepts surgissent qui matérialisent ces préoccupations; ils ont pour nom: disparité économique régionale, disparité sociale régionale, inégalité de développement, développement régional, planification, aménagement du territoire, décentralisation, régionalisation, etc. ${ }^{*(1)}$ Dès lors, le tourisme est promu comme outil privilégié de développement régional et s'insère dans ce discours. La littérature touristique abonde d'études de cas dans cette optique (Kemper, 1979) (Taylor, 1980) (Clarke, 1981) (Loukissas, 1982).

Le modèle de développement économique lié au tourisme est de plus en plus scruté et soumis à une évaluation serrée. Dans des analyses globales (Bodson. Stafford, 1983) (Schnell, 1974), comme dans des recherches à caractère méthodologique (Archer. 1972) sur l'effet multiplicateur ou l'incidence sur l'emploi par exemple, l'économique prime. Un auteur (Pearce, 1980) pour sa part, propose une évaluation basée sur une approche génétique tenant compte de la perspective historique.

\footnotetext{
- Daniel Dumas est bibhothecaire au Centre détudes du tourisme.
}

La gestion et la planification du tourisme en région posent des problèmes spécifiques que les chercheurs en tourisme ont tenté de circonscrire et de solutionner. D'une part, des modèles et des pratiques de planification et de développement (souvent liés à l'aménagement) sont présentés (Gunn, 1972 et 1980) (Baud-Bovy, Lawson, 1977) (Nicholls, 1980) (Palmer, Probert, 1980). D'autre part, des questions plus précises retiennent l'attention: le produit (Ritchie, Zins, 1978), la promotion (Davis, Taylor, 1980), la prospective (Edgell, Seely, 1980).

Le tourisme comme facteur de développement, et donc d'aplanissement des inégalités régionales, se heurte naturellement au cas des régions à faible potentiel touristique (Buckley, Witt, 1985). Finalement le discours sur le développement régional, discours souvent tenu par la société globale. pose les questions d'impact social et culturel sur la communauté (Pizam, Acquaro, 1977) (une abondante littérature existé sur ces impacts), de participation réelle de la population locale (Loukissas, 1983) (Moulin, 1980), bref de l'écoute des "paroles régionales".

Les documents présentés, qui ne sont qu'une sélection et qui peuvent être complétés par d'autres lectures, sont tous disponibles pour prêt ou consultation au CET.

\section{Tourisme et développement régional}

Associstion intemationale d'experts scientifiques du tourisme, Le tourisme: élément de l'aménagement du territoire et facteur d'expansion régionale, Berne (Suisse), ed. Gurten, 1960, $248 \mathrm{p}$.

CLARKE, Alan, Coastal development in France: tourism as a tool for regional development, Annals of Tourism Research, vol. VIII, no 3, (1981), pp. 447-461.

DEFERT, Pierre, Le tourisme facteur de valorisation régionale. Recherche sociale, no 3 (janvier-février 1966), $63 \mathrm{p}$.

KEMPER, Robert $\mathrm{V}_{\text {., }}$ Tounism in Taos and Patzcuaro: a comparison of two approaches to regional development, Annals of Tourism Research, vol. VI, no 16 (jan/march 1979), pp. $91=110$,
LOUKISSAS, Philippas, V., Tourism's regiona/ development impacts: a comparative analysis of the Greek islands. Annals of Tourism Research, vol. 9, no 4 (1982), pp. 523-541.

TAYLOR, P.A. and M.R. CARTER, Using tourism in regional development: planning for tourism in Scotiand, in Tourism Planning and Development Issues, Washington, D.C.: George Washington University, 1980, pp. 295-310.

\section{Aspects économiques}

ARCHER, Brian H., A regional employment multiplier, La revue de tourisme, vol. 27, no 3 (juillet-septembre 1972), pp. 105-107.

ARCHER, Brian $\mathrm{H}$. and Christine B. OWEN, Towards a tourist regional mu/tiplier, Journal of Travel Research, vol. XI, no 2, Ifall 1972]. pp. $9-13$.

BODSON, Paul et Jean STAFFORD, Les dimensions économiques de l'aménagement' touristique, Téoros, vol. 2, no 3 loctobre 1983). pp. 20-23.

PEARCE, Douglas G., Tourism and regional development: a genetic approach. Annals of Tourism Research, vol. VII, no 1, (1980), pp. $69-82$

SCHNELL, Peter, Tourism as a means of improving the regional economic structure, in Tourism as a factor in national and regional development: proceedings of a meeting of the International Geographical Union's working Group on the Geography of Tourism and Recreation, (Peterborough, sept. 1974), pp. 72-80.

\section{Gestion et planification du tourisme en région}

BAUD-BOVY, Manuel and Fred LAWSON. Approaches to regional and national planning. in Tourism and Recreation Developement: a handbook of physical planning. Bodson (Mass.): CBI Publishing. 1977, pp. 131.144.

BRADEN, Paul V, and Louise WIENER, Bringing travel, tourism and cultural resource activities in harmony with Regional economic development, in Tourism Marketing and Management Issues, Washington, D.C.: George Washington University, 1980, pp. 33-42.

BUCKLEY, P.J, and Stephen F. WITT, Tourism in difficult areas: case studies of Bradford. Bristol, Glasgow and Hamm, Tourism Managoment, vol. 6, no 3, (septembre 1985), pp. 206-214. 
DAVIS, K. Pascal and Bernard W. TAYLOR, $A_{4}$ goal programming model for allocating state promotional effort to regional markers in accordance with tourism potentiel, Journal of Travel Pesearch, vol, XVIII, no 4 (Spring 1900), pp. 24-30.

EDGELL, David and Richard SEELY, A multistage model for the development of interna. tional rourism forecasts for States and Regrons, in Tourism Planning and Development lissues, Washington, D.C.: George Washington University, 1980 , pp. 407-410.

GUNN, Clare A., An approach to regional assessment of tourism development potential, in Tourism Planning and Development lssues, Washington, D.C.; George Washington University, $1980, \mathrm{Pp}, 261-276$

GUNN, Clare A., Vacationscape: designing tourist regions. Austin (Texas): University of Texas, $1972,238 \mathrm{p}$
NiCHOLLS, Leland, L., Regional Tourism deve lopment in "Third World Americaw: a propo sed model for Appalachia, in Tourism Planning and Development issuess, Washington, D.C. George Washington University, 1980, pp 283-294

PALMER, John and Gordon PROBERT, Planning at the county level: a case study of Gwent, South Wales, Tourism Management. vol. 1, no 3, isept. 1980), pp. 158-167.

RITCHIE, J.R. Brent and Michel ZINS, Culture as determinant of the attractiveness of a tou rism region, Annals of Tourism Research, vol. $\checkmark$ no 2 (april/june 1978), pp. 252-267.

\section{Population et intéréts régionaux}

KNOPP, Timothy B., Tourism, the local interests and the function of public lands, in Tou-

rism Planning and Development Issues. Washington, D.C.: George Washington University, 1980, pp, 225238.

LOUKISSAS, P.J., Public participation in com munity tounism planning a gaming simulation approach, Journal of Travel Research, wol. XXII, ho 1 (summer 1983). pp. 18-23.

MOULiN, Claude, Plan for ecological and culfural rourism involwing particioation of loca! population and associations, in Tourism Planning and Development issues. Washington. D.C: George Washington University, 1990 , pp. 199-212.

PIZAM, Abraharn and Ernest J. ACQUARD, Some social costs and nebefits of tourism to rural communities: the Cape Cod case. Amherst (Mass. I: Massachusetts Agricultural Experiment Station, 1977, 84 p. $f$

gue refont les gestes attendus d'eux et calqués sur les modèles sans au préalable tenter de se définir et de se développer en fonction des besoins de leur région et de leur population

L'Abitibi-Témiscamingue a une spécificité touristique qui requiert, pour être exploitée de façon rentable au profit des résidents, qu'on s'y attarde et qu'on la développe. Dans ce pays neuf, depuis peu éveillé à l'idée que le tourisme peut y trouver place. la tentation est forte et le danger est grand de chercher à ressembler aux autres et de développer un produit touristique sur les mémes bases que les autres régions, en utilisant leurs paramètres, en suivant leur modèle.
L'Abitibi-Témiscamingue porte le poids du déséquilibre entre les grands centres et la périphérie, entre les grands enjeux économiques et le bien-être de la population, entre le tourisme générateur de retombées économiques et le tourisme générateur de santé sociale. Loin de choisir et de se situer face à ces grands enjeux, elle se met en file et quête sa part du gâteau. Et si l'avenir passait par une autre voie... $f$

\section{Adeferences}

(1) Gouvernement du Quebec, Batir le Qubbec. Enonce de politique economique, 1979, p. 411 .

(2) Idem, p. 412

(3) Document interne de la Direction de Fanalvse et du developpement. Ministere du Tourisme, 3 juin 1985.
(Suite de la page 35)

\section{Le développement et I'aménagement touristiques}

touristique régionale du Pays-de-L'Érable lors de l'élaboration du plan de développement touristiqué régional et sont actuellement pris en compte dans les schémas d'aménagement des MRC concernées.

À l'heure de la réalisation des projets reliês au couloir Beauce-Appalaches, peut-on espérer que le contrat de développement sera la formule idéale pour soutenir le développement touristique? Nous serons sûrement en mesure d'ici quelques mois de vérifier si l'objectif de cette formule a èté rempli. Ainsi, suite à l'expérimentation de la formule des accords-cadres de développement, 1'OPDQ devra remettre un rappor d'évaluation au gouvernement avant l'élaboration d'accords-cadres additionnels.

La Loi sur l'aménagement et l'urbanisme aura donc permis de susciter une nouvelle mentalité ouverte à la planification et à la concertation non pas seulement du milieu municipal mais aussi de plusieurs autres intervenants socio-économiques.

L'expérience des plans de développement touristique, pour leur part, nous démontre l'intérêt đu milieu municipal face à la planification de développement touristique régional par la participation de leur(s) représentant(s) au Comité de gestion.

Les dix contrats de développement qui seront préparés prochainement nous permettent de connaître davantage le degré de volonté des $\mathrm{MRC}$ de s'impliquer dans le développement régional. Par ailleurs, les deux (2) contrats de développement touchant, entre autres, le tourisme permettront de constater si la cohésion entre les intervenants politiques et socio-économiques est possible

La planification tant du développement tou ristique que de l'aménagement est donc en devenir; l'étal actuel de la situation nous permet d'être optimiste quant à la poursuite du processus.

Par contre, après l'etape de la planification, on devra passer à la mise en application des plans et schémas. Cette cohésion, qui a été amorcée depuis, peu, se poursuivra-t-elle? $\mathrm{L} a$ volonté de concertation et de conciliation des intervenants sera à ce moment déterminante $f$

\section{Rofbrences}

(11) Gouvernement du Quebec: Batir le Qubbec. Enance de politique "economique, 1979 Pp. $411-412$.

(2) Idem

(3) Gouvemement du Quebec: Le tourisme au Que. bec. 1984, р. 9 .

(4) GRAVEL. Robert, La crbation des MRC et la dbcentralisation gouvernementale: analyse et dvaluation, ENAP, 1983, p. 33

(5) Gouvernement du Guebec: Le choin des regions. 1983. D. 80 .

(6) OHfice de planification et de developpement du Quebec: Le contrat de devaloppement, 1985. p. 1. idocument peesente lors du Congress de I'Union des municipalites regionales de cornte et des municipalités locales du Quebee? 